\title{
Modeling and Control of Co-generation Power Plants: A Hybrid System Approach
}

\author{
Giancarlo Ferrari-Trecate ${ }^{1}$, Eduardo Gallestey ${ }^{2}$, Paolo Letizia ${ }^{1}$, \\ Matteo Spedicato ${ }^{1}$, Manfred Morari ${ }^{1}$, and Marc Antoine ${ }^{3}$ \\ 1 Institut für Automatik, \\ ETH - Swiss Federal Institute of Technology, ETL, \\ CH-8092 Zürich, Switzerland, \\ Tel. +41-1-6327812, Fax +41-1-6321211 \\ \{ferrari,letizia, spedicat,morari\}@aut.ee.ethz.ch \\ 2 ABB Corporate Research Ltd. \\ eduardo.gallestey@ch.abb.com \\ 3 ABB Power Automation Ltd. \\ marc.antoine@ch.abb.com
}

\begin{abstract}
In this paper the optimization of a combined cycle power plant is accomplished by exploiting hybrid systems, i.e. systems evolving according to continuous dynamics, discrete dynamics, and logic rules. The possibility of turning on/off the gas and steam turbine, the operating constraints (minimum up and down times) and the different types of start up of the turbines characterize the hybrid behavior of a combined cycle power plant. In order to model both the continuous/discrete dynamics and the switching between different operating conditions we use the framework of Mixed Logic Dynamical systems. Next, we recast the economic optimization problem as a Model Predictive Control (MPC) problem, that allows us to optimize the plant operations by taking into account the time variability of both prices and electricity/steam demands. Because of the presence of integer variables, the MPC scheme is formulated as a mixed integer linear program that can be solved in an efficient way by using commercial solvers.
\end{abstract}

\section{Introduction}

In the last decade, the electric power industry has been subject to deep changes in structure and organization. From the technological side, the use of combined cycle power plants (CCPP) became more and more popular because of their high efficiency. A typical CCPP is composed of a gas cycle and a steam cycle. The gas cycle is fed by fuel and produces electric power through the expansion of the gas in the gas turbine; the steam cycle is supplied with the output exhaust gas from the gas turbine and generates both electricity and steam for the industrial processes. From the economic side, the liberalization of the energy market promoted the need of operating CCPPs in the most efficient way, that is by maximizing the profits due to the sales of steam and electricity and by minimizing the operating costs. 
In this paper we consider the problem of optimizing the short-term operation of a CCPP, i.e. to optimize the plant on an hourly basis over a time horizon that may vary from few hours to one day [23]. A large stream of research in the power systems area focused on this problem. The usual recipe is to recast the economic optimization into the minimization of a cost functional and to account for the physical model of the plant through suitably defined constraints. The results available in the literature differ both in the features of the CCPP modeled and in the scope of optimization.

In [23], 13], 24] the CCPP is assumed in a standard operating condition and optimal scheduling of the resources is performed via non linear programming techniques. The main limitation is that the possibility of turning on/off the turbines is not considered and therefore it is not possible to determine the optimal switching strategy. The discrete features of a CCPP (i.e. the fact that turbines can be turned on/off, the minimum up and down time constraints and the priority constraints in start up sequences) can be captured by using binary decision variables along with continuous-valued variables describing physical quantities (e.g. mass, energy and flow rates).

In 21] binary variables are introduced to model the on/off status of the devices and the corresponding optimization problem is solved through the use of genetic algorithms. The same modeling feature is considered in [16] where the automatic computation of the optimal on/off input commands (fulfilling also operational priority constraints) is accomplished through Mixed Integer Linear Programming (MILP). However in both papers, the modeling of the CCPP is done in an ad-hoc fashion and the generalization to plants with different topologies and/or specifications seems difficult. Moreover, other important features such as minimum up and down times or the behavior during start up are neglected. A fairly complete model of a thermal unit, using integer variables for describing minimum up/down time constraints, ramp constraints and different startup procedures, is given in [2]. The behaviour of the unit is then optimized by solving MILP problems. Even if this approach could be adapted for modeling a single turbine of a CCPP, no methodological way for describing the coordination between different turbines is provided.

The aim of this paper is to show how both the tasks of modeling and optimization of CCPPs can be efficiently solved by resorting to hybrid system methodologies. Hybrid systems recently have attracted the interest of many researchers, because they can capture in a single model the interaction between continuous and discrete-valued dynamics. Various models for hybrid system have been proposed [18, [20], 66 and the research focused on the investigation of basic properties such as stability [5], [17, controllability and observability [3], and the development of control [4] [20], state estimation [11] and verification, [1] schemes.

We will use discrete-time hybrid systems in the Mixed Logical Dynamical (MLD) form [4] for two reasons. First, they provide a general framework for modeling many discrete features of CCPPs, including the coordination and prioritization between different devices; second, they are suitable to be used in on-line optimization schemes [4]. 
In Section [2 we briefly recall the basic features of MLD systems and in Section 3 we describe the CCPP plant we consider (the "Island" CCPP). In Section 3.2 it is shown how to model in the MLD form both the continuous and discrete features of the plant. The operation optimization is then described in Section 4 . We show how to recast the economic optimization problem in a Model Predictive Control (MPC) scheme for MLD systems that can be solved via Mixed Integer Liner Programming (MILP). The use of piecewise affine terms in the cost functional allows us to consider various economic factors as the earnings due to selling of the electric power and steam, the fixed running costs, the start up costs and the cost due to aging of plant components. Finally in Section 5 the most significant control experiments are illustrated and in Section 5.1 the computational burden of the optimization procedure is discussed.

\section{Hybrid Systems in the MLD Form}

The derivation of the MLD form of a hybrid system involves basically three steps [4]. The first one is to associate with a logical statement $S$, that can be either true or false, a binary variable $\delta \in\{0,1\}$ that is 1 if and only if the statement holds true. Then, the combination of elementary statements $S_{1}, \ldots, S_{q}$ into a compound statement via the Boolean operators $\operatorname{AND}(\wedge), \operatorname{OR}(\vee), \operatorname{NOT}(\sim)$ can be represented as linear inequalities over the corresponding binary variables $\delta_{i}, i=1, \ldots, q$.

An example would be the condition $a^{T} x \leq 0$ :

$$
\left[a^{T} x \leq 0\right] \Leftrightarrow[\delta=1]
$$

where $x \in X \subseteq R^{n}$ is a continuous variable and $X$ is a compact set. If one defines $m$ and $M$ as lower and upper bounds on $a^{T} x$ respectively, the inequalities

$$
\left\{\begin{array}{l}
a^{T} x \leq M-M \delta \\
a^{T} x \geq \varepsilon+(m-\varepsilon) \delta
\end{array}\right.
$$

assign the value $\delta=1$ if and only if the value of $x$ satisfies the threshold condition. Note that $\varepsilon>0$ is a small tolerance (usually close to the machine precision) introduced to replace the strict inequalities by non-strict ones.

The second step is to represent the product between linear functions and logic variables by introducing an auxiliary variable $z=\delta a^{T} x$. Equivalently, $z$ is uniquely specified through the mixed integer linear inequalities

$$
\left\{\begin{array}{l}
z \leq M \delta \\
z \geq m \delta \\
z \leq a^{T} x-m(1-\delta) \\
z \geq a^{T} x-M(1-\delta)
\end{array}\right.
$$

The third step is to include binary and auxiliary variables in an LTI discretetime dynamic system in order to describe in a unified model the evolution of the continuous and logic components of the system. 
The general MLD form of a hybrid system is [4]

$$
\begin{aligned}
x(t+1) & =A x(t)+B_{1} u(t)+B_{2} \delta(t)+B_{3} z(t) \\
y(t) & =C x(t)+D_{1} u(t)+D_{2} \delta(t)+D_{3} z(t) \\
E_{2} \delta(t) & +E_{3} z(t) \leq E_{1} u(t)+E_{4} x(t)+E_{5}
\end{aligned}
$$

where $x=\left[\begin{array}{ll}x_{c}^{T} & x_{l}^{T}\end{array}\right]^{T} \in R^{n_{c}} \times\{0,1\}^{n_{l}}$ are the continuous and binary states, $u=\left[\begin{array}{ll}u_{c}^{T} & u_{l}^{T}\end{array}\right]^{T} \in R^{m_{c}} \times\{0,1\}^{m_{l}}$ are the inputs, $y=\left[\begin{array}{ll}y_{c}^{T} & y_{l}^{T}\end{array}\right]^{T} \in R^{p_{c}} \times$ $\{0,1\}^{\hat{p}_{l}}$ the outputs, and $\delta \in\{0,1\}^{r_{l}}, z \in R^{r_{c}}$ represent auxiliary binary and continuous variables, respectively. All constraints on the states, the inputs, the $z$ and $\delta$ variables are summarized in the inequalities (1c). Note that, although the description (1a)-(1b)-(1c) seems to be linear, non linearity is hidden in the integrality constraints over the binary variables.

MLD systems are a versatile framework to model various classes of systems. For a detailed description of such capabilities we defer the reader to [4], 3]. The discrete-time formulation of the MLD system allows to develop numerically tractable schemes for solving complex problems, such as stability [7, state estimation [11], and control [4. In particular, MLD models were proven successful for recasting hybrid dynamic optimization problems into mixed-integer linear and quadratic programs solvable via branch and bound techniques.

In this paper, for the optimization of the plant we propose a predictive control scheme (Model Predictive Control - MPC) which is able to stabilize MLD systems on desired reference trajectories while fulfilling operating constraints.

In order to automatize the procedure for representing a hybrid system in the MLD form (1a)-(1b)-(1C), the compiler HYSDEL (HYbrid System DEscription Language), that generates the matrices of the MLD model starting from a highlevel description of the dynamic and logic of the system, was developed at ETH, Zürich [27].

\section{Hybrid Model of a Combined Cycle Power Plant}

The cogeneration combined cycle power plant Island consists of four main components: a gas turbine, a heat recovery steam generator, a steam turbine and a steam supply for a paper mill.

We adopted the simplified input/output description of the plant presented in 24. and represented in Figure 1. Note that the heat recovery steam generator does not appear in Figure 1 because it is hidden in the "steam turbine" block. The plant has two continuous-valued inputs $\left(u_{1}\right.$ and $\left.u_{2}\right)$, and two binary inputs $\left(u_{l 1}\right.$ and $\left.u_{l 2}\right)$ :

- $u_{1}$ is the set point for the gas turbine load (in percent). The permitted operation range for the gas turbine is in the interval $\left[u_{1, \min }, u_{1, \max }\right]$;

- $u_{2}$ is the steam mass flow to the paper mill. The permitted range for the steam flow is in the interval $\left[u_{2, \min }, u_{2, \max }\right]$; 


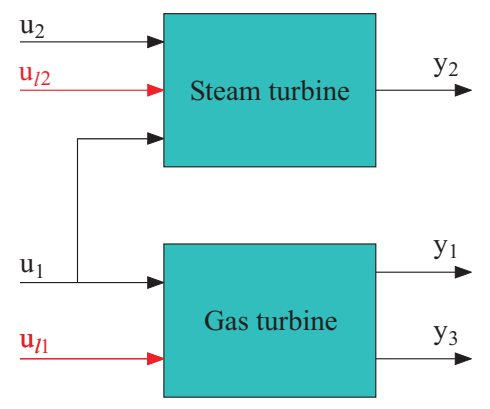

Fig. 1. Block diagram of the Island power plant.

- $u_{l 1}$ and $u_{l 2}$ are, respectively, the on/off commands for the gas and steam turbines; the "on" command is associated with the value one.

In the Island plant the inputs $u_{1}$ and $u_{2}$ are independent and all possible combinations within the admissible ranges are permitted. The binary input variables must fulfill the logic condition

$$
u_{l 2}=1 \quad \Rightarrow \quad u_{l 1}=1
$$

which defines a priority constraint between the two turbines: The steam turbine can be switched on/off only when the gas turbine is on, otherwise the steam turbine must be kept off.

The output variables of the model are:

- the fuel consumption of the gas turbine, $y_{1}[\mathrm{~kg} / \mathrm{s}]$;

- the electric power generated by the steam turbine, $y_{2}[\mathrm{MW}]$;

- the electric power generated by the gas turbine, $y_{3}[\mathrm{MW}]$;

Since we aim at optimizing the plant hourly, we chose a sampling time of one hour and we assume that the inputs are constant within each sampling interval. As reported in [24, an input/output model of the plant

$$
\begin{aligned}
& y_{1}(k+1)=f_{1}\left(u_{1}(k)\right) \\
& y_{2}(k+1)=f_{2}\left(u_{1}(k), u_{2}(k)\right) \\
& y_{3}(k+1)=f_{3}\left(u_{1}(k)\right)
\end{aligned}
$$

where the maps $f_{1}, f_{2}$ and $f_{3}$ can be either affine or piecewise affine and are obtained by interpolating experimental data. In particular, the use of piecewise affine input/output relations allows to approximate nonlinear behaviours in an accurate way.

\subsection{Hybrid Features of the Plant}

The features which suggest modeling the Island power plant as a hybrid system are the following: 
- the presence of the binary inputs $u_{l 1}$ and $u_{l 2}$;

- the turbines have different start up modes, depending on how long the turbines have been kept off;

- electric power, steam flow and fuel consumption are continuous valued quantities evolving with time.

Furthermore, the following constraints have to be taken into account:

- the operating constraints on the minimum amount of time for which the turbines must be kept on/off (the so-called minimum up/down times);

- the priority constraint (2). This condition, together with the previous one, leads to constraints on the sequences of logic inputs which can be applied to the system;

- the gas turbine load $u_{1}$ and the steam mass flow $u_{2}$ are bounded.

Finally one would also like to describe the piecewise affine relations (3)-(15) in the model of the CCPP.

\subsection{The MLD Model of the Island Plant}

All the features of the Island power plant mentioned in Section 3.1 can be captured by a hybrid model in the MLD form. For instance, the possibility to incorporate piecewise affine relations in the MLD model is discussed in [4]3] and the modeling of priority constraints like (2) is detailed in [4. Moreover the possibility of incorporating bounds on the inputs is apparent from the inequalities (1c). In the following we show, as an example, how to derive the MLD description of the different types of start up for the turbines. We focus on the steam turbine only, since the procedure is exactly the same for the gas turbine.

Typical start up diagrams show that the longer the time for which a turbine is kept off, the longer the time required before producing electric power when it is turned on. This feature can be modeled, in an approximate way, as a delay between the time instant when the command on is given and the instant when the production of electric power begins.

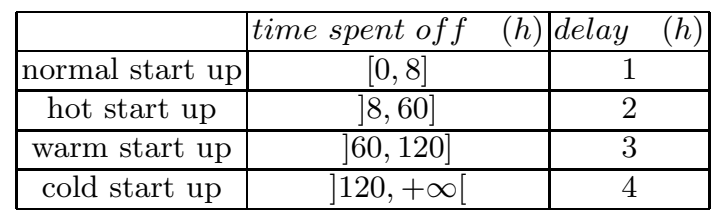

Table 1. Types of start up procedures for the steam turbine

In our model we consider the four different types of start up procedures for the steam and gas turbines, that are reported in Table 1, In order to take into account in the MLD model the different start up procedures, it is necessary to introduce three clocks with reset (which are state variables), five auxiliary logic variables $\delta$, and three auxiliary real variables $z$. 
The clocks are defined as follows:

- $\xi_{\text {on }}$ stores the consecutive time during which the turbine produces electric power. If the turbine is producing electric power, $\xi_{\text {on }}$ is increased according to the equation

$$
\xi_{\text {on }}(k+1)=\xi_{\text {on }}(k)+1
$$

otherwise it is kept equal to zero;

- $\xi_{\text {off }}$ stores the consecutive time during which the turbine does not produce electric power. So, if the turbine is off or does not produce electric power (as in a start up phase), $\xi_{\text {off }}$ is increased according to the equation

$$
\xi_{\text {off }}(k+1)=\xi_{\text {off }}(k)+1
$$

otherwise it is kept equal to zero;

$-\xi_{d}$, when it is positive, stores the delay that must occur between the turning on command and the actual production of electric power. If the turbine is turned on, $\xi_{d}$ starts to decrease according to the law

$$
\xi_{d}(k+1)=\xi_{d}(k)-1
$$

and the energy generation will begin only when the condition $\xi_{d}<0$ is fulfilled. Otherwise, if the turbine is off, $\xi_{d}$ stores the delay corresponding to the current type of start up. In view of Table 1] the value of $\xi_{d}$ is given by the following rules:

$$
\text { if } u_{l 2}=0 \text { and if } \begin{cases}\xi_{\text {off }} \leq 8 h & \Rightarrow \xi_{d}=0 \\ 8 h<\xi_{\text {off }} \leq 60 h & \Rightarrow \xi_{d}=1 \\ 60 h<\xi_{\text {off }} \leq 120 h & \Rightarrow \xi_{d}=2 \\ \xi_{\text {off }}>120 h & \Rightarrow \xi_{d}=3\end{cases}
$$

The procedure of deriving the MLD form of the clocks $\xi_{\text {on }}$, $\xi_{\text {off }}$ and $\xi_{d}$ by introducing auxiliary logic $(\delta)$ and real $(z)$ variables with the corresponding mixed-integer linear inequalities is reported in 26]. Clocks with reset in an MLD form are also discussed in [10].

The complete MLD model, capturing all the hybrid features of the Island plant described in Section 3.1, involves 12 state variables, $25 \delta$-variables and 9 $z$-variables [26]. The 103 inequalities stemming from the representation of the $\delta$ and $z$ variables are collected in the matrices $E_{i}, i=1, \ldots, 5$ of (1c) and are not reported here due to the lack of space. Some significant simulations which test the correctness of the MLD model of the Island power plant are also available in [26].

\section{Plant Optimization}

The control technique we use to optimize the operation of the Island power plant is the Model Predictive Control (MPC) [22], [4]. The main idea of MPC is to use 
a model of the plant (the MLD model in our case) to predict the future evolution of the system within a fixed prediction horizon. Based on this prediction, at each time step $k$ the controller selects a sequence of future command inputs through an on-line optimization procedure, which aims at minimizing a suitable cost function, and enforces fulfillment of the constraints. Then, only the first sample of the optimal sequence is applied to the plant at time $k$ and at time $k+1$, the whole optimization procedure is repeated. This on-line "re-planning" provides the desired feedback control action.

Economic optimization is achieved by designing the inputs of the plant that minimize a cost functional representing the operating costs. The terms composing the cost functional we consider are described in Section 4.1. In particular, some terms appearing in the cost functional are naturally non linear and in Section 4.2 we will show how to recast them into a linear form by using suitably defined auxiliary optimization variables. This allows reformulating the MPC problem as a Mixed Integer Linear Programming (MILP) problem, for which efficient solvers exist 12 .

\subsection{Cost Functional}

The following cost functional is minimized

$$
\begin{aligned}
J & =C_{\text {dem }}+C_{\text {change }}+C_{\text {fuel }}+C_{\text {start up }}+ \\
& +C_{\text {fixed }}-E+C_{\text {start up gas }}+C_{\text {fixed gas }}
\end{aligned}
$$

Let $k$ and $M$ be respectively the current time instant and the length of the control horizon. We use the notation $f(t \mid k)$ for indicating a time function, defined for $t \geq k$, that depends also on the current instant $k$. Then, the terms appearing in (10) have the following meaning:

$-C_{d e m}$ is the penalty function for not meeting the electric and steam demands over the prediction horizon:

$$
\begin{aligned}
C_{d e m}= & \sum_{\substack{t=k \\
k+M-1}}^{k+M-1} k_{d e m ~ e l}(t \mid k)\left|y_{2}(t \mid k)+y_{3}(t \mid k)-d_{e l}(t \mid k)\right|+ \\
& +\sum_{t=k}^{k+M} k_{d e m ~ s t}(t \mid k)\left|u_{2}(t \mid k)-d_{s t}(t \mid k)\right|
\end{aligned}
$$

where $k_{d e m ~ e l}(t \mid k)$ and $k_{d e m ~ s t}(t \mid k)$ are suitable positive weight coefficients; $d_{e l}(t \mid k)$ and $d_{s t}(t \mid k), t=k, \ldots, k+M-1$ represent, respectively, the profile of the electric and steam demands within the prediction horizon. Both the coefficients and the demands are assumed to be known over the prediction horizon. In actual implementation they are usually obtained by economic forecasting. The values of $k_{d e m ~ e l}(t \mid k)$ and $k_{d e m ~ s t}(t \mid k)$ weigh the fulfillment of the electric power demand and the fulfillment of the steam demand, respectively. 
- $C_{\text {change }}$ is the cost for changing the operation point between two consecutive time instants:

$$
\begin{gathered}
C_{\text {change }}=\sum_{t=k}^{k+M-2} k_{\Delta u_{1}}(t \mid k)\left|u_{1}(t+1 \mid k)-u_{1}(t \mid k)\right|+ \\
+\sum_{t=k}^{k+M-2} k_{\Delta u_{2}}(t \mid k)\left|u_{2}(t+1 \mid k)-u_{2}(t \mid k)\right|
\end{gathered}
$$

where $k_{\Delta u_{1}}(t \mid k), k_{\Delta u_{2}}(t \mid k)$ are the positive weights.

- $C_{\text {fuel }}$ takes into account the cost for fuel consumption (represented in the model by the output $y_{1}$ ).

$$
C_{\text {fuel }}=\sum_{t=k}^{k+M-1} k_{\text {fuel }}(t \mid k) y_{1}(t \mid k)
$$

where $k_{f u e l}(t \mid k)$ is the price of the fuel.

- $C_{\text {start up }}$ is the cost for the start up of the steam turbine. In fact, during the start up phase, no energy is produced and an additional cost related to fuel consumption is paid. $C_{\text {start up }}$ is then given by

$$
C_{\text {start up }}=\sum_{t=k}^{k+M-2} k_{\text {start up }}(t \mid k)\left(\max \left\{\left[u_{l 1}(t+1 \mid k)-u_{l 1}(t \mid k)\right], 0\right\}\right.
$$

where $k_{\text {start up }}(t \mid k)$ represents the positive weight coefficient. Note that

$$
\max \left\{\left[u_{l 1}(t+1 \mid k)-u_{l 1}(t \mid k)\right], 0\right\}
$$

is equal to one only if the start up of the steam turbine occurs, otherwise it is always equal to zero. Since, as discussed in Section 3.2 , different start up modes are allowed, $k_{\text {start up }}(t \mid k)$ should increase as the delay between the "on" command and the production of electric power increases (see Table 1).

- $C_{\text {fixed }}$ represents the fixed running cost of the steam turbine. It is non zero only when the device is on and it does not depend on the level of the steam flow $u_{2} . C_{\text {fixed }}$ is given by

$$
C_{\text {fixed }}=\sum_{t=k}^{k+M-1} k_{\text {fixed }}(t \mid k) u_{l 1}(t \mid k)
$$

where $k_{\text {fixed }}$ represents the fixed cost (per hour) due to the use of the turbine. Note that $C_{\text {fixed }}$ causes the steam turbine to be turned on only if the earnings by having it running are greater than the fixed costs.

- $E$ represents the earnings from the sales of steam and electricity; this term has to take into account that the surplus production can not be sold:

$$
\begin{aligned}
& E=\sum_{t=k}^{k+M-1} p_{e l}(t \mid k)\left(\min \left[y_{2}(t \mid k)+y_{3}(t \mid k), d_{e l}(t \mid k)\right]\right)+ \\
& \sum_{t=k}^{k+M-1} p_{s t}(t \mid k)\left(\min \left[u_{2}(t \mid k), d_{s t}(t \mid k)\right]\right)
\end{aligned}
$$


where $p_{e l}(t \mid k)$ and $p_{s t}(t \mid k)$ represent, respectively, the prices for electricity and steam.

- $C_{\text {start up gas }}$ is the start up cost for the gas turbine. It plays the same role as the term $C_{\text {start up }}$ and is defined through the logic input $u_{l 2}$. $C_{\text {start up gas }}$ is given by

$$
C_{\text {start up gas }}=\sum_{t=k}^{k+M-2} k_{\text {start up gas }}(t \mid k) \max \left\{\left[u_{l 2}(t+1 \mid k)-u_{l 2}(t \mid k)\right], 0\right\}
$$

where $k_{\text {start up gas }}(t \mid k)$ is a positive weight.

- $C_{\text {fixed gas }}$, represents the fixed running cost of the gas turbine (is analogous to $\left.C_{\text {fixed }}\right)$ :

$$
C_{\text {fixed gas }}=\sum_{t=k}^{k+M-1} k_{\text {fixed gas }}(t \mid k) u_{l 2}(t \mid k)
$$

where $k_{\text {fixed gas }}(t \mid k)$ is a positive weight.

\subsection{Constraints and Derivation of the MILP}

The constraints of the optimization problem are the system dynamics expressed in the MLD form (1a)-(1c). Thus, the overall optimization problem can be written as

$\min \quad J$

subject to $x(k \mid k)=x_{k}$ and for $t=k, \ldots, k+M$

$$
\begin{aligned}
& x(t+1 \mid k)=A x(t \mid k)+B_{1} u(t \mid k)+B_{2} \delta(t \mid k)+B_{3} z(t \mid k) \\
& y(t \mid k)=C x(t \mid k)+D_{1} u(t \mid k)+D_{2} \delta(t \mid k)+D_{3} z(t \mid k) \\
& E_{2} \delta(t \mid k)+E_{3} z(t \mid k) \leq E_{1} u(t \mid k)+E_{4} x(t \mid k)+E_{5}
\end{aligned}
$$

where the state $x_{k}$ of the system at time $k$ enters through the constraint $x(k \mid k)=x_{k}$ and the optimization variables are $\{u(t \mid k)\}_{t=k}^{k+M-1},\{\delta(t \mid k)\}_{t=k}^{k+M-1}$, $\{z(t \mid k)\}_{t=k}^{k+M-1}$.

In the following, for a signal $p(t \mid k)$ we denote with $\underline{p}_{k}$ the vector

$$
\underline{p}_{k}=[p(k \mid k) \cdots p(k+M-1 \mid k)]^{\prime} .
$$

Then, the optimization problem (12) can be written as follows:

$\min \quad J$

subject to $x(k \mid k)=x_{k}$ and for $t=k, \ldots, k+M$

$$
\begin{aligned}
& \underline{x}_{k+1}=T_{x} x_{k}+T_{u} \underline{u}_{T k}+T_{\delta} \underline{\delta}_{k}+T_{z} \underline{z}_{k} \\
& \underline{y}_{k}=C_{C} \underline{x}_{k+1}+D_{D_{1}} \underline{u}_{k}+D_{D_{2}} \underline{\delta}_{k}+D_{D_{3}} \underline{z}_{k}+\widetilde{C} x_{k} \\
& E_{E_{2}} \underline{\delta}_{k}+E_{E_{3}} \underline{z}_{k} \leq E_{E_{1}} \underline{u}_{k}+E_{E_{4}} \underline{x}_{k+1}+E_{E_{5}},
\end{aligned}
$$


where the entries of matrices $T_{x}, T_{u}, T_{\delta}$ and $T_{z}$ can be computed by successive substitutions involving the equation

$$
x(t)=A^{t-k} x_{k}+\sum_{i=0}^{t-k-1} A^{i}\left[B_{1} u(t-1-i)+B_{2} \delta(t-1-i)+B_{3} z(t-1-i)\right]
$$

that gives the state evolution of the MLD system. Also the $E_{E_{i}}$ matrices, $i=$ $1, \ldots, 5$ can be found by exploiting (15).

The optimization problem (14) is a mixed integer nonlinear program because of the nonlinearities appearing in the terms $C_{d e m}, C_{\text {change }}, C_{\text {start up }}, E$ and $C_{\text {start up gas. }}$. However the non linearities in the cost functional are of a special type. In fact, both the absolute value appearing in $C_{d e m}$ and $C_{\text {change, }}$, and the min / max functions appearing in $E, C_{\text {start up }}$ and $C_{\text {start up gas }}$ are piecewise affine maps and the optimization of a piecewise affine cost functional subject to linear inequalities can be always formulated as an MILP by suitably introducing further binary and continuous optimization variables [9]. The case of the cost functional $J$ is even simpler because, by using the fact that all the weight coefficients are positive, it is possible to write $J$ as a linear function of the unknowns without increasing the number of binary optimization variables. For details we defer the reader to 8,19 .

\section{Control Experiments}

In this section, we demonstrate the effectiveness of the proposed optimization procedure through some simulations.

The input/output equations describing the plant are given by (3)-(5) where

$$
\begin{aligned}
f_{1}\left(u_{1}\right) & =0.0748 \cdot u_{1}+2.0563 \\
f_{2}\left(u_{1}, u_{2}\right) & =0.62 \cdot u_{1}-0.857 \cdot u_{2}+29.714 \\
f_{3}\left(u_{1}\right) & =1.83 \cdot u_{1}-0.0012
\end{aligned}
$$

The permitted range for $u_{1}$ and $u_{2}$ are summarized in Table 2 For the Island plant, the affine models (16) and (18) are sufficiently accurate [24], whereas equation (17) is a crude approximation of the nonlinear behaviour and has a maximum error of $2 \%$. We highlight again the fact that a more precise MLD

\begin{tabular}{||l||l||l||}
\hline \hline Input & Minimum & Maximum \\
\hline \hline$u_{1}$ & $50 \%$ & $100 \%$ \\
\hline \hline$u_{2}$ & $2 \mathrm{~kg} / \mathrm{s}$ & $37 \mathrm{~kg} / \mathrm{s}$ \\
\hline \hline
\end{tabular}

Table 2. Upper and lower bounds on the inputs 
model could be obtained by using more accurate (and complex) piecewise affine approximations for the function $f_{2}$.

For a specified profile of the electric and steam demands, the optimizer chooses the optimal inputs in order to track the demands and at the same time minimize the operating costs. In particular the performance of the control action can be tuned by using suitable values of the weight coefficients appearing in the cost functional $J$. For example, the fulfillment of the electric power demand can be enforced in spite of the fulfillment of the steam demand if the ratio $k_{\text {dem el }} / k_{\text {dem st }}$ is high enough. In fact, due to the typical running of a combined cycle power plant, high values of both electric power and steam mass flow cannot be produced simultaneously because, in order to fulfill an high power demand, part of the steam must be used for running the steam turbine and cannot be supplied to the paper mill.

The turbines are switched on/off respecting the operating constraints (the minimum up and down times and the different types of start up). The use of different start up procedures implies that a minimum prediction horizon of five hours should be adopted in order to enable each type of start up. In fact the prediction horizon should be longer than the maximum delay that can occur between the command "on" and the production of electric power (which is four hours for a cold start up).

The control experiment we present was conducted over four days and a prediction horizon $M$ of 24 hours was adopted. The profile of the electric demand is a scaled version of the one reported in the "IEEE reliability test" [15. As is apparent from Figure 2(a) it has the feature that the demand during the week end differs from the one during the working days. Moreover the electricity prices are chosen proportionally to the profile of the electricity demand. The steam demand is constant and assumed to be near to the maximum level that can be generated by the plant (see Figure 3(b)].

Different start up costs for different start up procedures have been used. As remarked in Section 4.1, this can be done by properly choosing the weight coefficients $k_{\text {start up }}$ and $k_{\text {start up gas. }}$. The admissible values for $k_{\text {start up }}$ and $k_{\text {start up gas }}$ are summarized in Table 3 In order to illustrate how the startup coefficients are assigned, we focus on the gas turbine, being the procedure analogous for the steam turbine. If, at time $k$, the gas turbine is off, the type of startup is determined by the value of the counter $\xi_{d}$ (see formula (9)). Then, the numerical values of $k_{\text {start up }}(t \mid k), t=k, \ldots, k+M-1$, are determined according to Table 3 and by assuming that only one startup will occur in the control horizon. For instance, if $t_{o f f}(k)=60$ an "hot startup" should occur if the turbine is turned on in the next hour and a "warm startup" should occur if the turbine is tuned on in the next 60 houres. Then, if $M<60$, the values $k_{\text {start up }}(k \mid k)=58$ and $k_{\text {start up }}(t \mid k)=115, t=k+1, \ldots, k+M-1$ are used for determining the optimal inputs at time $k$. This guarantees that at least the first startup of the turbine within the control horizon is correctly penalized. 


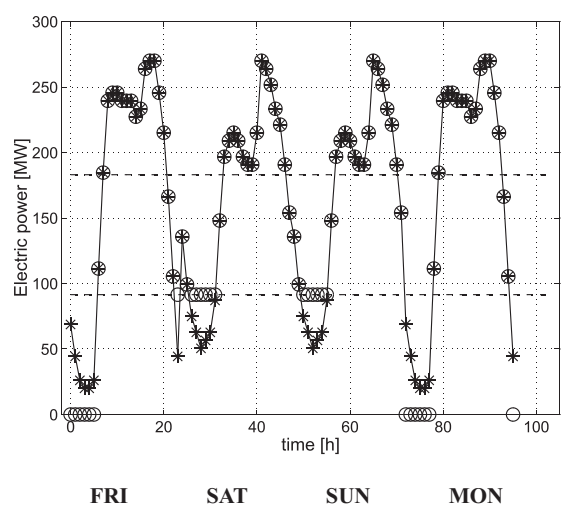

(a) Electric power demanded (stars and solid line) and produced by both the turbines (circles).

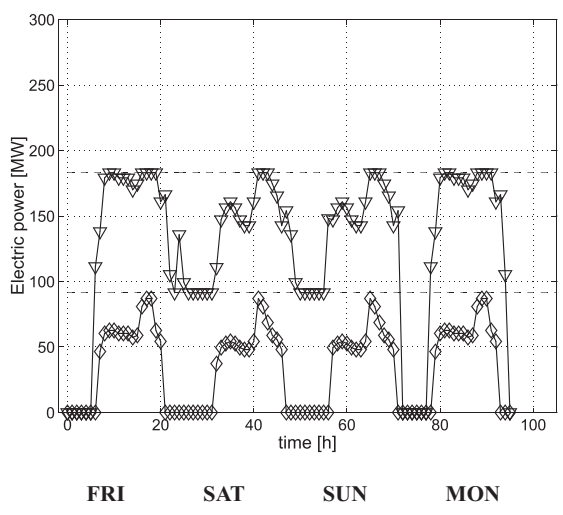

(b) Electric power produced by the steam turbine (diamonds) and by the gas turbine (triangles).

Fig. 2. Control experiment over 4 days with $M=24$ hours. The horizontal dashed line represents the maximum and minimum electric power that can be produced by the gas turbine.

\begin{tabular}{||l||l||l||}
\hline \hline NORMAL start up & $k_{\text {start up }}=30$ & $k_{\text {start up gas }}=30$ \\
\hline \hline HOT start up & $k_{\text {start up }}=58$ & $k_{\text {start up } \text { gas }}=58$ \\
\hline \hline WARM start up & $k_{\text {start up }}=115$ & $k_{\text {start up gas }}=115$ \\
\hline \hline COLD start up & $k_{\text {start up }}=152$ & $k_{\text {start up gas }}=152$ \\
\hline \hline
\end{tabular}

Table 3. Weights for the startup of the gas and steam turbines

The other weight coefficients have the constant values

$$
\begin{aligned}
& k_{\text {dem el }}=20[\mathrm{MW}], k_{\text {fuel }}=0.02\left[\frac{\mathrm{kg}}{\mathrm{s}}\right], k_{\text {dem st }}=1\left[\frac{\mathrm{kg}}{\mathrm{s}}\right], k_{\text {fixed }}=1 \text {, } \\
& k_{\Delta u_{1}}=0.001, \quad k_{\text {fixed gas }}=1, \quad k_{\Delta u_{2}}=0.001, \quad p_{s t}=0.2
\end{aligned}
$$

Note, in particular, that the fulfillment of the electric demand has an higher priority than the fulfillment of the steam demand because it holds $k_{\text {dem el }} \gg$ $k_{\text {dem st }}$.

At time $k=0$ the two turbines are assumed to have been off for one hour.

By looking at the electric power produced by each turbine (depicted in Figure 2(b) and the sequence of logic inputs (Figure 3(a) , one notes that at early morning of Friday and Monday the gas turbine is kept off because the demand is significantly below the minimum level that can be produced by the plant (the dashed line in Figure 2(a). On the other hand, during Friday and Saturday night, the gas turbine is kept on because the drop in demand is not big enough. From Figures 2(b) and 3(a) it is also apparent that the steam turbine is turned on when required. 


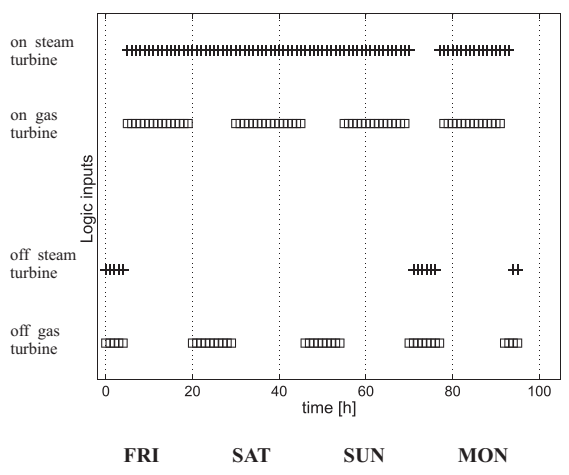

(a) Logic input of the gas turbine (squares) and of the steam turbine.

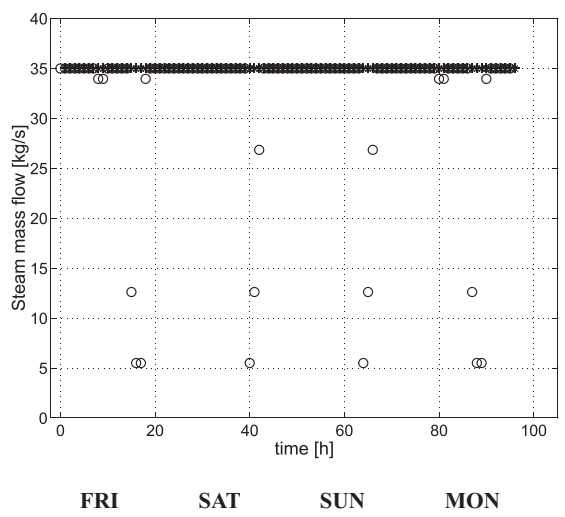

(b) Steam demanded (stars) and supplied (circles).

Fig. 3. Control experiment over 4 days with $M=24$ hours.

\subsection{Computational Complexity}

It is well known that MILP problems are NP-complete and their computational complexity strongly depends on the number of integer variables [25]. Therefore, the computational burden must be analyzed in order to decide about possibility of optimizing the CCPP on-line.

We considered the case study reported in Section 5, by using different prediction horizons $M$. At every time instant, an MILP problem with $(46 \cdot M-4)$ optimization variables $((27 \cdot M)$ of which are integer $)$, and $(119 \cdot M-8)$ mixed integer linear constraints was solved. The computation times (average and worst cases) needed for solving the MILPs, on a Pentium II-400 (running Matlab 5.3 for building the matrices defining the MILP and running CPLEX for solving it) are reported in Table 4

\begin{tabular}{||l||l||l||}
\hline \hline$M$ & Average times $[\mathrm{s}]$ & Worst case times $[\mathrm{s}]$ \\
\hline \hline 2 & 0.7705 & 0.8110 \\
\hline \hline 3 & 1.1335 & 1.2720 \\
\hline \hline 5 & 2.0996 & 4.4860 \\
\hline \hline 9 & 4.7323 & 9.7040 \\
\hline \hline 24 & 33.6142 & 101.7370 \\
\hline \hline
\end{tabular}

Table 4. Computational times for solving the MILP (14)

Note that the computation times increase as the prediction horizon $M$ becomes longer. However, the solution to the optimization problem took at most $102 \mathrm{~s}$, a time much shorter than the sampling time of one hour. 


\section{Conclusions}

The main goal of this paper is to show that hybrid systems in the MLD form provide a suitable framework for modeling CCPPs. In particular, many features like the possibility of switching on/off the turbines, the presence of minimum up and down times, priority constraints between turbines and different startup procedures can be captured by an MLD model. We point out that also other characteristics, like ramp constraints or nonlinear input/output relations (approximated by piecewise affine functions), can be easily incorporated in the MLD description.

Then, the optimization of the operation can be recasted into an MPC problem that can be efficiently solved by resorting to MILP solvers. The economic factors we considered in the definition of the cost functional are not the only possible choices. In fact different piecewise affine terms, reflecting other performance criteria could be added without changing the structure of the resulting optimization problem [9]. For instance the asset depreciation due to plant aging can be incorporated by exploiting lifetime consumption models [14].

\section{References}

1. R. Alur, T. A. Henzinger, and P. H. Ho. Automatic symbolic verification of embedded systems. IEEE Trans. on Software Engineering, 22(3):181-201, March 1996.

2. J.M. Arroyo and A.J. Conejo. Optimal response of a thermal unit to an electricity spot market. IEEE Trans. on Power Systems, 15(3):1098-1104, 2000.

3. A. Bemporad, G. Ferrari-Trecate, and M. Morari. Observability and Controllability of Piecewise Affine and Hybrid Systems. IEEE Trans. on Automatic Control, 45(10):1864-1876, 2000.

4. A. Bemporad and M. Morari. Control of systems integrating logic, dynamics, and constraints. Automatica, 35(3):407-427, 1999.

5. M.S. Branicky. Multiple Lyapunov functions and other analysis tools for switched and hybrid systems. IEEE Trans. on Automatic Control, 43(4):475-482, 1998.

6. M.S. Branicky, W.S. Borkar, and S.K. Mitter. A unified framework for hybrid control: model and optimal control theory. IEEE Trans. on Automatic Control, 43(1):31-45, 1998.

7. G. Ferrari-Trecate, F.A. Cuzzola, D. Mignone, and M. Morari. Analysis and control with performance of piecewise affine and hybrid systems. Proc. American Control Conference, pages 200-205, 2001.

8. G. Ferrari-Trecate, E. Gallestey, P. Letizia, M. Spedicato, M. Morari, and M. Antoine. Modeling and control of co-generation power plants: A hybrid system approach. Technical report, AUT01-18, Automatic Control Laboratory, ETH Zurich, 2001.

9. G. Ferrari-Trecate, P. Letizia, and M. Spedicato. Optimization with piecewiseaffine cost functions. Technical report, AUT00-13, Automatic Control Laboratory, ETH Zurich, 2001.

10. G. Ferrari-Trecate, D. Mignone, D. Castagnoli, and M. Morari. Mixed Logic Dynamical Model of a Hydroelectric Power Plant. Proceedings of the 4 th International Conference: Automation of Mixed Processes: Hybrid Dynamic Systems ADPM, Dortmund, Germany, 2000. 
11. G. Ferrari-Trecate, D. Mignone, and M. Morari. Moving horizon estimation for hybrid systems. IEEE Trans. on Automatic Control, 2002. to appear.

12. R. Fletcher and S. Leyffer. A mixed integer quadratic programming package. Technical report, Department of Mathematics, University of Dundee, Scotland, U.K., 1994.

13. C. A. Frangopoulos, A. I. Lygeros, C. T. Markou, and P. Kaloritis. Thermoeconomic operation optimization of the Hellenic Aspropyrgos Refinery combined-cycle cogeneration system. Applied Thermal Engineering, 16(12):949-958, 1996.

14. E. Gallestey, A. Stothert, M. Antoine, and S. Morton. Model predictive control and the optimisation of power plant load while considering lifetime consumption. IEEE Trans. on Power Systems, 2001. To appear.

15. C. Grigg and P. Wong. The IEEE Reliability Test System-1996. IEEE Trans. on Power Systems, 14(3):1010-1020, August 1999.

16. K. Ito and R. Yokoyama. Operational strategy for an industrial gas turbine cogeneration plant. International Journal of Global Energy Issues, 7(3/4):162-170, 1995.

17. M. Johannson and A. Rantzer. Computation of piecewise quadratic Lyapunov functions for hybrid systems. IEEE Trans. on Automatic Control, 43(4):555-559, 1998.

18. G. Labinaz, M.M. Bayoumi, and K. Rudie. A Survey of Modeling and Control of Hybrid Systems. Annual Reviews of Control, 21:79-92, 1997.

19. P. Letizia. Controllo di impianti cogenerativi mediante sistemi ibridi, 2001. M. Sc. thesis, Universita' degli Studi di Pavia.

20. J. Lygeros, C. Tomlin, and S. Sastry. Controllers for reachability specifications for hybrid systems. Automatica, 35(3):349-370, 1999.

21. D. A. Manolas, C. A. Frangopoulos, T. P. Gialamas, and D. T. Tsahalis. Operation optimization of an industrial cogeneration system by a genetic algorithm. Energy Conversion Management, 38(15-17):1625-1636, 1997.

22. M. Morari, J. Lee, and C. Garcia. Model Predictive Control. Prentice Hall, Draft Manuscript, 2001.

23. K. Moslehi, M. Khadem, R. Bernal, and G. Hernandez. Optimization of multiplant cogeneration system operation including electric and steam networks. IEEE Trans. on Power Systems, 6(2):484-490, 1991.

24. K. Mossig. Load optimization. Technical report, ABB Corporate Research, Baden (Zurich), 2000.

25. G.L. Nemhauser and L.A. Wolsey. Integer and Combinatorial Optimization. Wiley, 1988.

26. M. Spedicato. Modellizzazione di impianti cogenerativi mediante sistemi ibridi, 2001. M. Sc. thesis, Universita' degli Studi di Pavia.

27. F. D. Torrisi, A. Bemporad, and D. Mignone. HYSDEL - A Tool for Generating Hybrid Models. Technical report, AUT00-03, Automatic Control Laboratory, ETH Zurich, 2000. 